\section{Prospective evidence discourages secondary cytoreductive surgery}

For decades, patients with recurrent ovarian cancer have undergone secondary cytoreductive surgery (SCS). "The prevailing opinion, based on retrospective series and included in the National Comprehensive Cancer Network guidelines, was that surgery should be considered in selected patients," summarizes lead investigator Robert Coleman. The results of the prospective randomized controlled GOG-0213 trial now indicate that SCS followed by chemotherapy does not improve overall survival (OS) over chemotherapy alone.

"We designed GOG-0213 to answer two questions: does the addition of bevacizumab improve the effect of paclitaxel and carboplatin chemotherapy, and does secondary surgery improve survival outcomes," explains Coleman. Women with platinum-sensitive recurrent epithelial ovarian cancer were randomly assigned to SCS followed by platinumbased chemotherapy $(n=240)$ or chemotherapy alone $(n=245)$.

At a median follow-up duration of 48.1 months, "the median OS with surgery borders on being almost harmful," Coleman opines. Indeed, median OS durations were 50.6 months and 64.7 months with SCS and chemotherapy alone, respectively (HR 1.29, 95\% CI $0.97-1.72 ; P=0.08)$.

Compared with incomplete resection, complete gross resection was associated with longer median OS durations: 56.0 months versus 37.8 months (HR 0.61, 95\% CI 0.40-0.93). Complete gross resection did not, however, result in an OS benefit compared with chemotherapy alone: 56.0 months versus 64.7 months (HR 1.03, 95\% CI 0.74-1.46).

"We estimated OS durations of 22 months when we wrote the trial

protocol in 2000! The difference with the currently reported OS durations is proof of impressive advances in overall care for these patients, including better chemotherapy regimens," Coleman notes.

Patient-reported quality of life scores were significantly lower immediately after SCS than with chemotherapy alone, but no significant differences were reported at later time points. Three deaths (one in the SCS group and two in the chemotherapy alone group) were deemed to be treatment-related.

GOG- 0213 provides prospective evidence that patients with recurrent ovarian cancer do not derive an additional OS benefit from SCS before receiving chemotherapy. The results of three randomized surgery trials in this disease setting (DESKTOP III, with $O S$ as the primary end point, and SOC 1 and SOCceR, with progressionfree survival as the primary end point) are eagerly awaited.

with surgery

borders on

being almost harmful

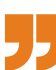

IMMUNOTHERAPY

\title{
MSI-H: a truly agnostic biomarker?
}

The FDA accelerated approvals of pembrolizumab for patients with microsatellite instability-high (MSI-H)/ deficient DNA mismatch repair (dMMR) metastatic or unresectable solid tumours, and of nivolumab (with or without ipilimumab), for patients with MSI-H/dMMR metastatic colorectal cancer (CRC) provide potentially curative options for such patients. Now, data from KEYNOTE-164 and KEYNOTE-158 provide further evidence on the efficacy of pembrolizumb in this setting.

In KEYNOTE-164, a total of 124 patients with metastatic MSI-H/dMMR CRC were assigned to either group $\mathrm{A}$ (on the basis of receipt of $\geq 1$ prior line of therapy) or group B ( $\geq 2$ prior lines) and received pembrolizumab for up to 35 cycles or until disease progression. The primary end point was objective response rate (ORR).

At a median follow-up duration of 31.3 months (group A) and 24.2 months (group B), both groups had an ORR of $33 \%$. Median overall survival (OS) durations were 31.4 months in group $A$ and not reached in group $B$ (2-year OS $63 \%$ ), with similar risks of grade 3-4 treatment-related adverse events (TRAEs, $16 \%$ and $13 \%$, respectively).

In KEYNOTE-158, a total of 233 patients with one of 27 different advanced-stage MSI-H/dMMR nonCRC solid tumour types received 3-weekly pembrolizumab for up to 2 years or until disease progression, with a primary end point of ORR.

At a median follow-up duration of 13.4 months, $33.4 \%$ of patients had an objective response; however, ORRs varied substantially when stratified by primary tumour histology. Among tumour types for which $>10$ patients were included, those with endometrial cancer had an ORR of $57.1 \%$, compared with $18.2 \%$ for pancreatic cancer and $0 \%$ for CNS cancers. These lower ORRs were reflected in median $O S$ durations of 4.0 months and 5.6 months among patients with pancreatic cancer or glioblastoma, respectively. By contrast,

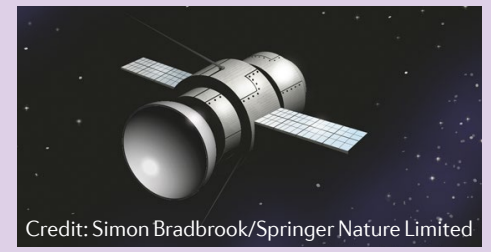

median OS was not reached in the endometrial, gastric, ovarian, or small intestine cancer subgroups and was 24.3 months among patients with cholangiocarcinoma.

Similar to KEYNOTE-164, 14.6\% of patients had grade 3-4 TRAEs. One patient died of treatment-related pneumonia.

These findings support the continued use of immune-checkpoint inhibitors in the majority of patients with MSI-H/ dMMR solid tumours. However, the substantially lower ORRs and OS durations of patients with pancreatic or CNS cancers challenge the idea that MSI-H/ dMMR can be applied to all solid tumour types regardless of primary histology.

Peter Sidaway

ORIGINAL ARTICLES Le, D. T. et al.J. Clin. Oncol. https://doi.org/10.1200/JCO.19.02107 (2019)| Marabelle, A. et al. J. Clin. Oncol. https://doi.org/ 10.1200/JCO.19.02105 (2019) 